\title{
Qualidade de pêssegos cv. “Eldorado” tratados com aminoetoxivinilglicina e ethephon e armazenados em atmosfera controlada
}

\author{
Quality of cv. 'Eldorado' peach fruit treated with aminoethoxyvinylglycine and ethephon and stored \\ in controlled atmosphere
}

\author{
Auri Brackmann ${ }^{\mathrm{I}}$ Ricardo Fabiano Hettwer GiehI ${ }^{\mathrm{II}}$ Ivan Sestari ${ }^{\mathrm{II}}$ \\ Josuel Alfredo Vilela Pinto ${ }^{\text {IV }}$ Ana Cristina Eisermann ${ }^{\text {IV }}$
}

\section{RESUMO}

\begin{abstract}
O objetivo desse trabalho foi avaliar o efeito da combinação da aplicação em pré-colheita de aminoetoxivinilglicina (AVG) e de ethephon com o armazenamento em atmosfera controlada (AC) na manutenção da qualidade e na redução de desordens causadas pela baixa temperatura em pêssegos $\mathrm{cv}$. "Eldorado", visando ao transporte marítimo. Os tratamentos constituíram-se da aplicação de AVG (125g ha $\mathrm{g}^{-1}$ e $250 \mathrm{~g} \mathrm{ha}^{-1}$ aos 15 ou 21 dias antes da colheita), de ethephon (140g ha-1 aos sete dias antes da colheita) e do controle (sem aplicação de produtos), combinados com duas condições de armazenamento em AC 1,0kPa de $\mathrm{O}_{2}+3,0 \mathrm{kPa}$ de $\mathrm{CO}_{2}$ e 2,0kPa de $\mathrm{O}_{2}+5,0 \mathrm{kPa}$ de $\mathrm{CO}_{2}$ a $0^{\circ} \mathrm{C}$. Após 24 dias de armazenamento em $A C$ e de mais dois e quatro dias a $15^{\circ} \mathrm{C}$, simulando o período médio de transporte marítimo até a Europa, observou-se que a aplicação de ethephon aumentou a produção de etileno e reduziu a acidez titulável e a firmeza da polpa dos frutos, acelerando o amadurecimento. A aplicação de AVG na pré-colheita não retardou o amadurecimento dos frutos, porém a aplicação $125 \mathrm{~g} \mathrm{ha}^{-1}$ de AVG aos 15 dias antes da colheita reduziu a incidência de escurecimento da polpa, em relação ao controle e ao ethephon. Além disso, a condição de atmosfera controlada com $2,0 \mathrm{kPa}$ de $\mathrm{O}_{2}+5,0 \mathrm{kPa}$ de $\mathrm{CO}_{2}$ permitiu maior acidez titulável e menor incidência de escurecimento da polpa.
\end{abstract}

Palavras-chave: Prunus persica (L.) Batsch., AVG, etileno, escurecimento da polpa.

\section{ABSTRACT}

The effect of preharvest application of aminoethoxyvinylglycine (AVG) and ethephon combined with controlled atmosphere (CA) storage conditions on peach fruit (cv. Eldorado) quality maintenance and chilling injury prevention was investigated, aiming at the maritime transport. Treatments evaluated were preharvest application of AVG (125g $\mathrm{ha}^{-1}$ and $250 \mathrm{~g} \mathrm{ha}^{-1}$ at 15 or 21 days before harvest), ethephon (140g ha 7 days before harvest), and control, combined with two CA conditions $\left(1.0 \mathrm{kPaO} \mathrm{O}_{2}+3.0 \mathrm{kPaCO}\right.$ and $2.0 \mathrm{kPaO} \mathrm{O}_{2}+$ $5.0 \mathrm{kPa} \mathrm{CO}$ at $0^{\circ} \mathrm{C}$ ). After 24 days of $\mathrm{CA}$ storage plus 2 and 4 days of shelf-life at $15^{\circ} \mathrm{C}$, preharvest application of ethephon stimulated fruit ripening by increasing ethylene production and decreasing both flesh firmness and titratable acidity. Application of AVG did not delay fruit ripening. However, fruit treated with $125 \mathrm{~g} \mathrm{ha}^{-1}$ of $A V G$ at 15 days before harvest showed lower incidence of internal browning than ethephon-treated and control fruit. Furthermore, storage of fruit under $C A$ with $2.0 \mathrm{kPaO} \mathrm{O}_{2}+5.0 \mathrm{kPa} \mathrm{CO}_{2}$ allowed higher titratable acidity and lower incidence of internal browning.

Key words: Prunus persica (L.) Batsch., AVG, ethylene, internal browning.

\section{INTRODUÇÃO}

O desenvolvimento de cultivares de pêssego com características que permitem sua comercialização para o consumo in natura e o processamento industrial, como a cultivar "Eldorado", tem possibilitado um aumento na área cultivada com pêssegos no Rio Grande do Sul. A cultivar "Eldorado" destaca-se por produzir frutos grandes, com $60 \%$ de cobertura vermeha, polpa amarelo-ouro e excelente

\footnotetext{
IDepartamento de Fitotecnia, Universidade Federal de Santa Maria (UFSM), 97105-900, Santa Maria, RS, Brasil. E-mail: brackman@ccr.ufsm.br. Autor para correspondência.

IIPrograma de Pós-graduação em Agronomia, UFSM, Santa Maria, RS, Brasil.

IIIPrograma de Pós-Graduação em Fisiologia e Bioquímica de Plantas da Escola Superior de Agricultura Luiz de Queiroz (ESALQ),

Universidade de São Paulo (USP), Piracicaba, SP, Brasil.

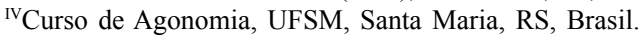


sabor (CERETTA et al., 2000). O aumento na oferta cria a possibilidade de parte da produção ser comercializada em outros Estados brasileiros ou de ser exportada, principalmente para a Europa. Dessa forma, técnicas que visam a reduzir o amadurecimento tornam-se necessárias para manter a qualidade dos frutos durante o transporte.

O pêssego é um fruto climatérico, apresentando modificações expressivas durante o amadurecimento, um processo complexo e programado geneticamente, que culmina na alteração da cor, da firmeza da polpa, do flavour e do aroma, estando o etileno envolvido no desencadeamento desses eventos (ALEXANDER \& GRIERSON, 2002). A aminoetoxivinilglicina (AVG; ácido [S]-trans-2-amino4-[2'-aminoetoxi]-3-butenóico) é um composto que se liga de forma reversível à ACC sintase (HUAI et al., 2001), impedindo a conversão de $\mathrm{S}$-adenosilmetionina para ácido 1-aminociclopropano-1-carboxílico (ACC), o precursor imediato na rota de biossíntese do etileno (KENDE, 1993). A aplicação pré-colheita de AVG em pêssegos cv. "Redhaven" e nectarinas cv. "Stark Red Gold" reduziu significativamente a produção de etileno e a perda da firmeza da polpa (BREGOLI et al., 2002; TORRIGIANI et al., 2004).

Pêssegos cv. "Eldorado" desenvolvem escurecimento interno quando armazenados sob baixas temperaturas, embora essa cultivar não seja muito sensível a danos por frio (CERETTA et al., 2000). O etileno, apesar dos efeitos drásticos na redução da vida pós-colheita, parece exercer um papel importante na prevenção e/ou redução de desordens causadas por baixas temperaturas em pêssegos e nectarinas. A presença de etileno na câmara de armazenamento reduziu a incidência de dano por frio em nectarinas cv. "Flavortop" armazenadas a $0^{\circ} \mathrm{C}$, enquanto a aplicação de 1-metilciclopropeno, um inibidor da ação do etileno, aumentou a ocorrência desses distúrbios (DONG et al., 2001). Dessa forma, pêssegos tratados com AVG poderiam ficar mais sensíveis à incidência de desordens fisiológicas causadas pelo armazenamento sob baixas temperaturas. Por outro lado, a aplicação pré-colheita de ethephon (ácido 2-cloroetilfosfônico), um composto que libera etileno quando hidrolisado, poderia atuar na prevenção do dano por frio, um efeito ainda não estudado. Outra alternativa é o armazenamento em atmosfera controlada, que reduz a ocorrência de danos por frio em nectarinas e pêssegos, além de manter melhor qualidade físico-química (ZHOU et al., 2000; CERETTA et al., 2000; NAVA \& BRACKMANN, 2002).

O objetivo deste trabalho foi avaliar o efeito da combinação da aplicação de AVG e de ethephon com o armazenamento em AC na manutenção da qualidade e na redução de desordens causadas pela baixa temperatura em pêssegos cv. "Eldorado", visando ao o transporte marítimo por um período de 24 dias.

\section{MATERIAL E MÉTODOS}

O experimento foi conduzido no Núcleo de Pesquisa em Pós-Colheita do Departamento de Fitotecnia da Universidade Federal de Santa Maria, no ano agrícola de 2003/2004. Os tratamentos pré-colheita foram realizados em pessegueiros do pomar comercial Santo Antônio, da empresa Firpo, localizado no município de Canguçu, RS.

A aplicação de AVG foi realizada com um pulverizador costal com capacidade para 20L. O volume de calda aplicado foi de aproximadamente $1.000 \mathrm{~L} \mathrm{ha}^{-1}$. Utilizou-se como fonte de AVG o produto comercial ReTain $^{\circledR}$ (15\% de ingrediente ativo). O produto comercial Ethrel $^{\circledR}$ foi utilizado como fonte de ethephon ( $24 \%$ de ingrediente ativo). Além disso, empregou-se, juntamente com o AVG e o ethephon, o espalhante adesivo organosiliconado Silwet $\mathrm{L} 77^{\circledR}$ na concentração de $0,05 \%(\mathrm{v} / \mathrm{v})$.

A aplicação dos produtos foi efetuada em unidades experimentais com cinco plantas, sendo usadas quatro repetições por tratamento. Destas, foram colhidos apenas os frutos das três plantas centrais para o armazenamento. $\mathrm{O}$ delineamento experimental utilizado foi o inteiramente casualizado em esquema bifatorial, com quatro repetições por avaliação, contendo 15 frutos cada. Os tratamentos consistiram da combinação dos fatores doses e épocas de aplicação de produtos e condições de AC. Para o primeiro fator,

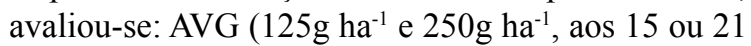
dias antes da colheita), ethephon ( $140 \mathrm{~g} \mathrm{ha}^{-1}$ aos sete dias antes da colheita) e controle (sem aplicação de produtos); e, para o segundo fator, as condições de armazenamento em AC avaliadas foram $1,0 \mathrm{kPa}$ de $\mathrm{O}_{2}+$ $3,0 \mathrm{kPa}$ de $\mathrm{CO}_{2}$ e $2,0 \mathrm{kPa} \mathrm{de} \mathrm{O}_{2}+5,0 \mathrm{kPa}$ de $\mathrm{CO}_{2}$ a $0^{\circ} \mathrm{C}$.

As condições de $\mathrm{AC}$ foram instaladas em minicâmaras experimentais, com volume de 233L depois de estabilizada a temperatura de armazenamento, mediante a injeção, nas minicâmaras, de nitrogênio (N2) proveniente de um gerador de $\mathrm{N}_{2}$, que funciona pelo princípio "Pressure Swing Adsorption" (PSA). Através da diluição com $\mathrm{N}_{2}$, reduziu-se a pressão parcial do $\mathrm{O}_{2}$ até o nível preestabelecido para cada tratamento. As pressões parciais de $\mathrm{CO}_{2}$ foram obtidas mediante a injeção deste gás, proveniente de cilindros de alta pressão, no interior das minicâmaras.

As pressões parciais dos gases $\left(\mathrm{O}_{2}\right.$ e $\left.\mathrm{CO}_{2}\right)$, durante o período de armazenamento, foram monitoradas e corrigidas por meio de um equipamento 
totalmente automatizado da marca KronenbergerClimasul. A temperatura das câmaras frigoríficas foi regulada automaticamente através de termostatos eletrônicos. Além disso, foi monitorada diariamente através de termômetros de mercúrio com resolução de $0,1^{\circ} \mathrm{C}$ inseridos na polpa de alguns frutos.

As análises laboratoriais foram realizadas após 24 dias de armazenamento em atmosfera controlada a $0^{\circ} \mathrm{C}$ e mais dois e quatro dias a $15^{\circ} \mathrm{C}$, simulando o período de trânsito marítimo e de comercialização. Os parâmetros avaliados foram: produção de etileno, determinada por meio de cromatografia gasosa (cromatógrafo Varian ${ }^{\circledR}$, modelo CX3400), sendo os resultados expressos em $\mu \mathrm{L} \mathrm{kg}^{-1} \mathrm{~h}^{-1}$; respiração dos frutos, expressa em $\mathrm{mL} \mathrm{CO} \mathrm{kg}^{-1} \mathrm{~h}^{-1} \mathrm{e}$ determinada com o auxílio de um analisador de gases com fluxo contínuo (Agridatalog ${ }^{\circledR}$ ); firmeza da polpa, avaliada por meio de um penetrômetro com ponteira de 7,9mm de diâmetro e expressa em Newton; acidez titulável, obtida pela titulação, com $\mathrm{NaOH} 0,1 \mathrm{~N}$, de uma solução de $10 \mathrm{~mL}$ de suco em $100 \mathrm{~mL}$ de água destilada até atingir $\mathrm{pH} 8,1$; incidência de escurecimento da polpa, avaliada através da contagem dos frutos que apresentavam sintomas visuais característicos desse distúrbio; e incidência de podridões, pela contagem dos frutos que apresentavam lesões apodrecidas ( $>5 \mathrm{~mm}$ de diâmetro).
Os dados, expressos em porcentagem, foram transformados pela fórmula $\operatorname{arc}$.sen $\sqrt{x / 100}$, sendo submetidos, com os demais, à análise da variância. As médias foram comparadas entre si pelo teste de Duncan em nível de 5\% de probabilidade de erro.

\section{RESULTADOS E DISCUSSÃO}

De acordo com a análise da variância, os fatores tratamentos em pré-colheita (AVG e ethephon) e condições de AC não apresentaram interação significativa sobre os parâmetros de qualidade avaliados após 24 dias de armazenamento em $\mathrm{AC} \mathrm{a} 0^{\circ} \mathrm{C}$ e mais dois e quatro dias a $15^{\circ} \mathrm{C}$ (Tabelas 1,2 e 3 ). Esse resultado significa que, dentro das condições avaliadas, não há a necessidade de se optar por uma condição de AC específica em virtude de determinado tratamento pré-colheita realizado no pomar.

A produção de etileno foi maior nos frutos tratados com ethephon e significativamente menor nos frutos tratados com $125 \mathrm{~g} \mathrm{~L}^{-1}$ de AVG 15 dias antes da colheita, quando comparado ao controle, tanto aos dois quanto aos quatro dias a $15^{\circ} \mathrm{C}$ (Tabela 1). Efeito drástico do ethephon no aumento da produção de etileno foi observado por GUELFAT-REICH \& BENARIE (1975) em ameixas e damascos. Provavelmente o etileno liberado pelo ethephon estimulou a expressão de isoformas de ACC sintase responsáveis pela

Tabela 1 - Qualidade de pêssegos cv. "Eldorado" tratados na pré-colheita com AVG e ethephon e armazenados em atmosfera controlada durante 24 dias a $0^{\circ} \mathrm{C}$ e mais dois e quatro dias de exposição a $15^{\circ} \mathrm{C}$. Santa Maria, RS. 2004.

\begin{tabular}{|c|c|c|c|c|}
\hline Produto e dose $\left(\mathrm{g} \mathrm{ha}^{-1}\right)$ & Momento da aplicação & $\begin{array}{l}\text { Produção de etileno } \\
\qquad\left(\mu \mathrm{L} \mathrm{kg}^{-1} \mathrm{~h}^{-1}\right)\end{array}$ & \multirow{2}{*}{$\begin{array}{c}\text { Respiração } \\
\left(\mathrm{mL} \mathrm{CO}_{2} \mathrm{~kg}^{-1} \mathrm{~h}^{-1}\right) \\
\text { dias a } 20^{\circ} \mathrm{C}\end{array}$} & \multirow{2}{*}{$\begin{array}{c}\text { Firmeza da polpa } \\
\text { (N) }\end{array}$} \\
\hline & & & & \\
\hline \multicolumn{2}{|c|}{ Controle } & $4,99 \mathrm{~b}^{* *}$ & $30,26 \mathrm{a}$ & $65,7 \mathrm{a}$ \\
\hline AVG - 125 & $15 \mathrm{DAC}^{*}$ & $2,68 \mathrm{c}$ & $27,52 \mathrm{ab}$ & $68,3 \mathrm{a}$ \\
\hline AVG - 250 & $15 \mathrm{DAC}$ & $3,38 \mathrm{bc}$ & $24,66 \mathrm{~b}$ & $66,8 \mathrm{a}$ \\
\hline AVG - 125 & $21 \mathrm{DAC}$ & $4,48 \mathrm{bc}$ & $27,33 \mathrm{ab}$ & $70,1 \mathrm{a}$ \\
\hline AVG - 250 & $21 \mathrm{DAC}$ & $3,81 \mathrm{bc}$ & $26,54 \mathrm{ab}$ & $68,5 \mathrm{a}$ \\
\hline Ethephon - 140 & $7 \mathrm{DAC}$ & $9,14 \mathrm{a}$ & $29,29 \mathrm{a}$ & $54,4 \mathrm{~b}$ \\
\hline \multicolumn{2}{|c|}{$\mathrm{CV}(\%)$} & 30,45 & 11,11 & 11,19 \\
\hline & & \multicolumn{3}{|c|}{ Quatro dias a $20^{\circ} \mathrm{C}$} \\
\hline \multicolumn{2}{|c|}{ Controle } & $7,22 \mathrm{~b}$ & $30,05 \mathrm{a}$ & $53,2 \mathrm{a}$ \\
\hline AVG - 125 & $15 \mathrm{DAC}$ & $3,58 \mathrm{c}$ & $29,33 \mathrm{a}$ & $58,5 \mathrm{a}$ \\
\hline AVG - 250 & $15 \mathrm{DAC}$ & $4,09 \mathrm{c}$ & $28,11 \mathrm{a}$ & $55,5 \mathrm{a}$ \\
\hline AVG - 125 & $21 \mathrm{DAC}$ & $5,66 \mathrm{bc}$ & $31,46 \mathrm{a}$ & $51,4 \mathrm{a}$ \\
\hline AVG - 250 & $21 \mathrm{DAC}$ & $6,06 \mathrm{bc}$ & $30,85 \mathrm{a}$ & $51,5 \mathrm{a}$ \\
\hline Ethephon - 140 & $7 \mathrm{DAC}$ & $12,97 \mathrm{a}$ & $32,30 \mathrm{a}$ & $43,7 \mathrm{a}$ \\
\hline \multicolumn{2}{|c|}{$\mathrm{CV}(\%)$} & 36,08 & 21,40 & 10,69 \\
\hline
\end{tabular}

*DAC $=$ dias antes da colheita.

**Médias seguidas de mesma letra, na vertical e dentro de cada momento de avaliação, não diferem entre si pelo teste de Duncan em nível de $5 \%$ de probabilidade de erro. 
Tabela 2 - Qualidade de pêssegos cv. "Eldorado" tratados na pré-colheita com AVG e ethephon e armazenados em atmosfera controlada durante 24 dias a $0^{\circ} \mathrm{C}$ e mais dois e quatro dias de exposição a $15^{\circ} \mathrm{C}$. Santa Maria, RS. 2004.

\begin{tabular}{|c|c|c|c|c|}
\hline Produto e dose $\left(\mathrm{g} \mathrm{ha}^{-1}\right)$ & Momento da aplicação & \multirow{2}{*}{ Acidez titulável (meq 100mL $\mathrm{m}^{-1}$ ) } & \multirow{2}{*}{$\begin{array}{l}\text { Incidência podridões (\%) } \\
\text { Dois dias a } 20^{\circ} \mathrm{C}\end{array}$} & \multirow[t]{2}{*}{$\begin{array}{l}\text { Escurecimento da polpa } \\
\qquad(\%)\end{array}$} \\
\hline & & & & \\
\hline \multicolumn{2}{|c|}{ Controle } & $10,2 b^{* *}$ & $10,6 \mathrm{a}$ & $9,1 \mathrm{ab}$ \\
\hline AVG - 125 & $15 \mathrm{DAC}^{*}$ & $11,6 \mathrm{a}$ & $10,6 \mathrm{a}$ & $5,7 \mathrm{~b}$ \\
\hline AVG - 250 & $15 \mathrm{DAC}$ & $10,9 \mathrm{ab}$ & $3,9 \mathrm{a}$ & $3,3 \mathrm{~b}$ \\
\hline AVG - 125 & $21 \mathrm{DAC}$ & $10,9 a b$ & $14,1 \mathrm{a}$ & $13,8 \mathrm{a}$ \\
\hline AVG - 250 & $21 \mathrm{DAC}$ & $10,3 \mathrm{~b}$ & $8,2 \mathrm{a}$ & $5,4 \mathrm{~b}$ \\
\hline Ethephon - 140 & $7 \mathrm{DAC}$ & $9,4 \mathrm{c}$ & $6,7 \mathrm{a}$ & $12,3 \mathrm{ab}$ \\
\hline \multicolumn{2}{|c|}{ CV (\%) } & 5,71 & 53,94 & 50,00 \\
\hline & & \multicolumn{3}{|c|}{ Quatro dias a $20^{\circ} \mathrm{C}$} \\
\hline $\mathrm{Co}$ & role & $10,8 \mathrm{~b}$ & $32,6 \mathrm{a}$ & $32,9 \mathrm{a}$ \\
\hline AVG - 125 & $15 \mathrm{DAC}$ & $12,0 \mathrm{a}$ & $42,9 \mathrm{a}$ & $18,4 \mathrm{~b}$ \\
\hline AVG - 250 & $15 \mathrm{DAC}$ & $10,8 \mathrm{~b}$ & $24,3 \mathrm{a}$ & $23,9 a b$ \\
\hline AVG - 125 & $21 \mathrm{DAC}$ & $10,8 \mathrm{~b}$ & $41,1 \mathrm{a}$ & $20,1 \mathrm{~b}$ \\
\hline AVG - 250 & $21 \mathrm{DAC}$ & $10,7 \mathrm{~b}$ & $48,1 \mathrm{a}$ & $28,8 \mathrm{ab}$ \\
\hline Ethephon - 140 & $7 \mathrm{DAC}$ & $9,0 \mathrm{c}$ & $36,6 \mathrm{a}$ & $35,3 \mathrm{a}$ \\
\hline \multicolumn{2}{|c|}{$\mathrm{CV}(\%)$} & 7,81 & 32,96 & 22,70 \\
\hline
\end{tabular}

*DAC $=$ dias antes da colheita.

**Médias seguidas de mesma letra, na vertical e dentro de cada momento de avaliação, não diferem entre si pelo teste de Duncan em nível de $5 \%$ de probabilidade de erro.

produção autocatalítica desse hormônio, como observado por BARRY et al. (2000) em tomates. Por outro lado, a aplicação de AVG reduziu a produção de etileno de pêssegos cv. "Redhaven" e nectarinas cv. "Stark Red Gold" (BREGOLI et al., 2002; TORRIGIANI et al., 2004, respectivamente), pois esse composto inibe a atividade da ACC sintase (HUAI et al., 2001). Em pêssegos cv. "Eldorado", o presente estudo demonstrou que a época de aplicação e a dose de AVG não influenciam de forma significativa a produção de etileno. No entanto, TORRIGIANI et al. (2004) obtiveram melhor resultado com a aplicação de AVG aos 21 dias antes da colheita, em relação aos oito dias em nectarinas, possivelmente por inibir também as isoformas da ACC sintase expressas na transição entre o sistema 1 e 2 de síntese de etileno. As duas condições de AC não afetaram significativamente a produção de etileno, nas duas avaliações (Tabela 3). Em pêssegos cv. "Chiripá", a produção de etileno foi eficientemente reduzida com o armazenamento em AC com 2,0kPa de $\mathrm{O}_{2}+6-7 \mathrm{kPa}$ de $\mathrm{CO}_{2}$ (NAVA \& BRACKMANN, 2002). É importante salientar que a aplicação de ethephon provocou uma queda antecipada das folhas (dados não apresentados), um efeito provavelmente associado ao etileno liberado por esse composto (ALEXANDER \& GRIERSON, 2002).

O tratamento dos frutos com $250 \mathrm{~g} \mathrm{~L}^{-1} \mathrm{de}$ AVG 15 dias antes da colheita resultou em menor produção de $\mathrm{CO}_{2}$, em comparação ao tratamento controle e à aplicação de ethephon, aos dois dias a $15^{\circ} \mathrm{C}$ (Tabela 1). McGLASSON et al. (2005) verificaram menor respiração nos frutos tratados com AVG e colhidos sete dias após os frutos não-tratados, sendo que a colheita três dias após a aplicação não teve o mesmo efeito. Os frutos armazenados em AC com $2,0 \mathrm{kPa}$ de $\mathrm{O}_{2}+5,0 \mathrm{kPa}$ de $\mathrm{CO}_{2}$ apresentaram menor respiração após dois e quatro dias a $15^{\circ} \mathrm{C}$ (Tabela 3 ). Esses resultados podem ter ocorrido em função da pressão parcial de $\mathrm{CO}_{2}$ mais elevada, que pode inibir várias enzimas do ciclo dos ácidos tricarboxílicos, como a isocitrato desidrogenase (LIU et al., 2004).

A firmeza da polpa foi significativamente menor nos frutos tratados com ethephon nas avaliações realizadas após 24 dias de armazenamento em $\mathrm{AC}$ e mais dois e quatro dias a $15^{\circ} \mathrm{C}$ (Tabelas 1$)$. A aplicação de ethephon reduziu consideravelmente a firmeza da polpa de nectarinas e damascos (GUELFAT-REICH \& BEN-ARIE, 1975). O efeito drástico do ethephon na redução da firmeza da polpa deve ter ocorrido pelo estímulo da síntese de etileno nesses frutos (Tabelas 1), o qual atua na modulação da atividade de enzimas de degradação das paredes celulares, como algumas isoformas de poligalacturonases, $\beta$-galactosidases e expansinas (ALEXANDER \& GRIERSON, 2002). No presente estudo, não houve efeito significativo da aplicação de AVG sobre a retenção da firmeza da polpa, quando comparado ao controle (Tabelas 1), contrariamente ao 
Tabela 3 - Qualidade de pêssegos cv. "Eldorado" tratados na pré-colheita com AVG e ethephon e armazenados em atmosfera controlada durante 24 dias a $0^{\circ} \mathrm{C}$ e mais dois e quatro dias de exposição a $15^{\circ} \mathrm{C}$. Santa Maria, RS. 2004.

\begin{tabular}{|c|c|c|c|c|}
\hline \multirow{3}{*}{ Parâmetros } & \multicolumn{2}{|c|}{ Dois dias a $20^{\circ} \mathrm{C}$} & \multicolumn{2}{|c|}{ Quatro dias a $20^{\circ} \mathrm{C}$} \\
\hline & \multicolumn{2}{|c|}{$\mathrm{O}_{2}+\mathrm{CO}_{2}(\mathrm{kPa})$} & \multicolumn{2}{|c|}{$\mathrm{O}_{2}+\mathrm{CO}_{2}(\mathrm{kPa})$} \\
\hline & $1+3$ & $2+5$ & $1+3$ & $2+5$ \\
\hline Produção de etileno $\left(\mu \mathrm{L} \mathrm{kg}^{-1} \mathrm{~h}^{-1}\right)$ & $4,74 \mathrm{a}^{*}$ & $4,75 \mathrm{a}$ & $6,54 \mathrm{a}$ & $6,65 \mathrm{a}$ \\
\hline Respiração $\left(\mathrm{mL} \mathrm{CO} \mathrm{kg}^{-1} \mathrm{~h}^{-1}\right)$ & $29,85 \mathrm{a}$ & $25,35 \mathrm{~b}$ & $33,30 \mathrm{a}$ & $27,39 \mathrm{~b}$ \\
\hline Firmeza da polpa $(\mathrm{N})$ & $64,8 \mathrm{a}$ & $66,4 \mathrm{a}$ & $49,8 \mathrm{~b}$ & $54,7 \mathrm{a}$ \\
\hline Acidez titulável (meq $\left.100 \mathrm{~mL}^{-1}\right)$ & $10,3 \mathrm{a}$ & $10,7 \mathrm{a}$ & $10,1 \mathrm{~b}$ & $11,3 \mathrm{a}$ \\
\hline Incidência de podridões (\%) & $9,1 \mathrm{a}$ & 8,9 a & 39,9 a & $35,2 \mathrm{a}$ \\
\hline Escurecimento interno (\%) & $7,5 \mathrm{a}$ & $9,0 \mathrm{a}$ & $31,2 \mathrm{a}$ & $21,9 \mathrm{~b}$ \\
\hline
\end{tabular}

*Médias seguidas de mesma letra, na horizontal e dentro de cada momento de avaliação, não diferem entre si pelo teste de Duncan em nível de $5 \%$ de probabilidade de erro.

observado por TORRIGIANI et al. (2004) em nectarinas. A AC com $2,0 \mathrm{kPa}$ de $\mathrm{O}_{2}+5,0 \mathrm{kPa}$ de $\mathrm{CO}_{2}$ manteve a firmeza da polpa mais elevada após quatro dias a $15^{\circ} \mathrm{C}$ (Tabela 3). CERETTA et al. (2000) obtiveram melhor retenção da firmeza da polpa de pêssegos cv. "Eldorado" com o armazenamento em AC com 1,0kPa de $\mathrm{O}_{2}+3,0 \mathrm{kPa}$ de $\mathrm{CO}_{2}$, em relação a $1,5 \mathrm{kPa}$ de $\mathrm{O}_{2}+$ $5,0 \mathrm{kPa}$ de $\mathrm{CO}_{2}$, após 46 dias, enquanto que não observaram diferença significativa aos 32 dias.

A aplicação pré-colheita de $125 \mathrm{~g} \mathrm{~L}^{-1} \mathrm{de} \mathrm{AVG}$, 15 dias antes da colheita, proporcionou maior acidez titulável, nas duas avaliações (Tabela 2). As outras doses e épocas de aplicação de AVG não permitiram resultados superiores ao controle, para esse parâmetro, sendo que a aplicação de ethephon teve um efeito drástico na redução da acidez dos frutos (Tabela 2). TORRIGIANI et al. (2004) verificaram, no momento da colheita, maior acidez titulável em nectarinas cv. "Stark Red Gold" tratadas com AVG. A relação entre o etileno e o metabolismo dos ácidos orgânicos ainda não foi devidamente elucidada, porém ETIENNE et al. (2002) verificaram que a expressão de cDNAs para proteínaschave do metabolismo dos ácidos orgânicos é maior durante o amadurecimento, sendo concomitante com o aumento na produção de etileno. Os frutos armazenados em AC com 2,0kPa de $\mathrm{O}_{2}+5,0 \mathrm{kPa}$ de $\mathrm{CO}_{2}$ apresentaram maior acidez titulável ao final de quatro dias a $15^{\circ} \mathrm{C}$ (Tabela 3). Provavelmente, a redução na taxa respiratória dos frutos armazenados nessa condição (Tabela 1) resultou em menor consumo dos ácidos orgânicos no ciclo dos ácidos tricarboxílicos (ETIENNE et al., 2002).

Para o parâmetro incidência de podridões, não se observou diferença significativa, independentemente dos tratamentos avaliados (Tabelas 2 e 3). AL-MASRI et al. (2006) verificaram que a aplicação de ethephon promoveu o desenvolvimento de mofo-branco, causado por Sclerotinia sclerotiorum em feijoeiro e pepineiro, enquanto que o uso de $\mathrm{AVG}$ reduziu significativamente o crescimento micelial e o desenvolvimento desse fungo. Esse resultado foi atribuído à inibição da produção de etileno nos tecidos do fungo e/ou das plantas infectadas. No entanto, os resultados obtidos neste experimento não demonstraram esses mesmos efeitos sobre a incidência de podridões em pêssegos cv. "Eldorado", as quais são causadas principalmente por Monilinia fructicola. Segundo PALOU et al. (2003), a exposição de frutos de caroço a diferentes concentrações de etileno, durante o armazenamento, também não afetou a incidência e a severidade de podridões causadas por $\boldsymbol{M}$. fructicola.

A incidência de escurecimento interno foi menor nos frutos tratados com $125 \mathrm{~g} \mathrm{ha}^{-1}$ de AVG aos 15 dias antes da colheita, em comparação ao controle e ao uso de ethephon, tanto aos dois quanto como aos quatro dias a $15^{\circ} \mathrm{C}$ (Tabela 2). A aplicação de AVG em pêssegos cv. "Feicheng" reduziu o desenvolvimento de escurecimento interno (JU et al., 1999), mas aumentou a incidência de lanosidade em nectarinas $\mathrm{cv}$. "Artic Snow" (McGLASSON et al., 2005). Possivelmente o etileno não possui a mesma importância na prevenção do escurecimento interno como na redução de outros danos causados pelo frio, como a lanosidade (DONG et al., 2001). De acordo com LURIE \& CRISOSTO (2005), o escurecimento da polpa está relacionado a modificações na permeabilidade das membranas celulares, que podem permitir a interação dos compostos fenólicos com as polifenoloxidases. Por outro lado, a lanosidade é decorrente da falta de sincronia entre a atividade das poligalacturonases e as pectinaesterases (ZHOU et al., 2000). Apesar de não ter ocorrido diferença significativa entre tratamentos 
aos dois dias a $15^{\circ} \mathrm{C}$, após quatro dias nessas condições, a incidência de escurecimento interno foi menor nos frutos armazenados em AC com 2,0kPa de $\mathrm{O}_{2}+5,0 \mathrm{kPa}$ de $\mathrm{CO}_{2}$ (Tabela 3). CERETTA et al. (2000) observaram menor incidência de escurecimento em pêssegos cv. "Eldorado" armazenados em AC com $1,0 \mathrm{kPa}$ de $\mathrm{O}_{2}+3,0 \mathrm{kPa}$ de $\mathrm{CO}_{2}$ a $-0,5^{\circ} \mathrm{C}$, enquanto que a $0,5^{\circ} \mathrm{C}$ não houve diferença significativa entre as condições de AC avaliadas. Já em pêssegos cv. "Chiripá", a incidência de lanosidade foi reduzida com o armazenamento em AC com $1,0 \mathrm{kPa}$ de $\mathrm{O}_{2}+4,0 \mathrm{kPa}$ de $\mathrm{CO}_{2}$ e $2,0 \mathrm{kPa}$ de $\mathrm{O}_{2}+8,0 \mathrm{kPa}$ de $\mathrm{CO}_{2}$, após quatro meses a $-0,5^{\circ} \mathrm{C}$ (NAVA \& BRACKMANN, 2002).

\section{CONCLUSÕES}

A aplicação de $125 \mathrm{~g} \quad \mathrm{ha}^{-1}$ de aminoetoxivinilglicina 15 dias antes da colheita reduz a incidência de escurecimento da polpa de pêssegos cv. "Eldorado" durante a exposição a $15^{\circ} \mathrm{C}$, após 24 dias de armazenamento em atmosfera controlada a $0^{\circ} \mathrm{C}$, enquanto que o uso de ethephon acelera o amadurecimento. A condição de atmosfera controlada com $2,0 \mathrm{kPa}$ de $\mathrm{O}_{2}+5,0 \mathrm{kPa}$ de $\mathrm{CO}_{2}$ reduz o escurecimento da polpa sem afetar negativamente a qualidade físico-química dos frutos.

\section{AGRADECIMENTOS}

Ao Conselho Nacional de Desenvolvimento Científico e Tecnológico ( $\mathrm{CNPq})$, pelo financiamento de parte deste projeto e pela concessão de bolsas aos pesquisadores Giehl e Eisermann e à Fundação de Amparo à Pesquisa do Rio Grande do Sul (FAPERGS), pela concessão de bolsa ao pesquisador Pinto.

\section{REFERÊNCIAS}

ALEXANDER, L.; GRIERSON, D. Ethylene biosynthesis and action in tomato: a model for climacteric fruit ripening. Journal of Experimental Botany, Lancaster, v.53, n.377, p.2039-2055, 2002.

AL-MASRI, M.I. et al. Ethylene production by Sclerotinia sclerotiorum and influence of exogenously applied hormone and its inhibitor aminoethoxyvinylglycine on white mold. Crop Protection, Amsterdam, v.25, n.4, p.356-361, 2006.

BARRY, C.S. et al. The regulation of 1-aminocyclopropane-1carboxylic acid synthase gene expression during the transition from system-1 to system-2 ethylene synthesis in tomato. Plant Physiology, Lancaster, v.123, p.979-986, 2000.

BREGOLI, A.M. et al. Peach (Prunus persica) fruit ripening: aminoethoxyvinylglycine (AVG) and exogenous polyamines affect ethylene emission and flesh firmness. Physiologia Plantarum, Copenhagen, v.114, p.472-481, 2002.

CERETTA, M. et al. Conservação em atmosfera controlada de pêssego cultivar Eldorado. Ciência Rural, Santa Maria, v.30, n.1, p.73-79, 2000.
DONG, L. et al. Ethylene involvement in the cold storage disorder of 'Flavortop' nectarine. Postharvest Biology and Technology, Amsterdam, v.23, n.3, p.105-115, 2001.

ETIENNE, C. et al. Isolation and characterization of six peach cDNAs encoding key proteins in organic acid metabolism and solute accumulation: involvement in regulating peach fruit acidity. Physiologia Plantarum, Copenhagen, v.114, n.2, p.259-270, 2002.

GUELFAT-REICH, S.; BEN-ARIE, R. A comparison of the ripening effects of ethephon on Japanese plums and apricots. In: FACTEURS ET RÉGULATION DE LA MATURATION DES FRUITS, 238., 1975, Paris. Colloque... Paris: Centre National de la Recherche Scientifique, 1975. N.238, 368p. p. 105-108.

HUAI, Q. et al. Crystal structures of 1-aminocyclopropane-1carboxylate (ACC) synthase in complex with aminoethoxyvinylglycine and pyridoxal-5' - phosphate provide new insight into catalytic mechanisms. Journal of Biological Chemistry, Maryland, v.276, n.41, p.38210-38216, 2001.

JU, Z. et al. Combinations of GA3 and AVG delay fruit maturation, increase fruit size and improve storage life of 'Feicheng' peaches. Journal of Horticultural Science and Biotechnology, Ashford, v.74, p.579-583, 1999.

KENDE, H. Ethylene biosynthesis. Annual Review of Plant Physiology and Molecular Biology, Palo Alto, v.44, p.283307, 1993.

LIU, S. et al. Effects of $\mathrm{CO}_{2}$ on respiratory metabolism in ripening banana fruit. Postharvest Biology and Technology, Amsterdam, v.33, n.1, p.27-34, 2004.

LURIE, S.; CRISOSTO, C.H. Chilling injury in peach and nectarine. Postharvest Biology and Technology, Amsterdam, v.37, n.3, p.195-208, 2005.

McGLASSON, W.B. et al. Preharvest application of aminoethoxyvinylglycine (AVG) modifies harvest maturity and cool storage life of 'Arctic Snow' nectarines. Postharvest Biology and Technology, Amsterdam, v.36, n.1, p.93-102, 2005 .

NAVA, G.A.; BRACKMANN, A. Armazenamento de pêssegos (Prunus persica (L.) Batsch), cv. Chiripá, em atmosfera controlada. Revista Brasileira de Fruticultura, Jaboticabal, v.24, n.2, p.328-332, 2002.

PALOU, L. et al. Effect of continuous exposure to exogenous ethylene during cold storage on postharvest decay development and quality attributes of stone fruits and table grapes. Postharvest Biology and Technology, Amsterdam, v.27, n.3, p.243-254, 2003.

TORRIGIANI, $P$ et al. Pre-harvest polyamine and aminoethoxyvinylglycine (AVG) applications modulate fruit ripening in Stark Red Gold nectarines (Prunus persica L. Batsch). Postharvest Biology and Technology, Amsterdam, v.33, n.3, p.293-308, 2004.

ZHOU, H. W. et al. Delayed storage and controlled atmosphere storage of nectarines: two strategies to prevent woolliness. Postharvest Biology and Technology, Amsterdam, n.18, n.2, p. 133-141, 2000.

Ciência Rural, v.37, n.1, jan-fev, 2007. 